\title{
SÍNTOMAS COMPRESIVOS Y ASOCIACIÓN CON EL VOLUMEN DE LOS ESPECÍMENES QUIRÚRGICOS EN PACIENTES CON BOCIO
}

\author{
COMPRESSIVE SYMPTOMS AND THEIR ASSOCIATION WITH THE \\ VOLUME OF SURGICAL SPECIMENS IN PATIENTS WITH GOITER
}

Fernández-Delgado Alberto ${ }^{1}$

Osorio-Covo Carlos ${ }^{1}$

Herrera-Sáenz Francisco ${ }^{2}$

Correspondencia: fherreras@gruposcalpellum.com

Recibido para evaluación: marzo - 15 - 2015. Aceptado para publicación: octubre - 25 - 2015

\section{RESUMEN}

Introducción: es imperativa la necesidad de efectuar estudios que evalúen y describan si existe una asociación entre las características morfológicas del bocio, en especial el volumen y la manifestación de síntomas como ortopnea, disfagia y fonastenia.

Objetivo: evaluar en pacientes con bocio la prevalencia de síntomas compresivos (SC) y su asociación con el volumen de los especímenes quirúrgicos.

Materiales y métodos: mediante una entrevista realizada en el acto prequirúrgico, fueron evaluadas las manifestaciones de SC en pacientes con bocio y posteriormente, se calculó el volumen de cada espécimen quirúrgico. Se determinó la asociación existente entre SC y el volumen de los especímenes quirúrgicos.

Resultados: la población de estudio estuvo conformada por 92 pacientes, $86(93.5 \%)$ mujeres y $6(6.5 \%)$ varones. Edad promedio $46.1 \pm 14.9$ años. La prevalencia de síntomas fue: disfagia $(41.3 \%)$, ortopnea (39.1\%) y fonastenia $(12.0 \%)$. Tanto la ausencia de SC y el hallazgo ocasional se asociaron a volúmenes menores de 50 mililitros $(p<0.001)$. El hallazgo simultáneo de ortopnea y disfagia se asoció a volúmenes entre 50 y 99 mililitros $(p<0.001)$, por último, el hallazgo de fonastenia asociada a disnea y disfagia se asoció a volúmenes superiores a 100 mililitros $(p<0.001)$.

Conclusión: el volumen del espécimen quirúrgico estuvo asociado con el hallazgo de SC en el paciente con bocio. Rev.cienc.biomed. 2015;6(2):259-264

\section{PALABRAS CLAVE}

Bocio; Signos; Síntomas.

\section{SUMMARY}

Introduction: It is necessary to conduct clinical studies to evaluate and describe if there is an association between the morphological characteristics of goiter, especially its volume, and the manifestation of symptoms such as orthopnea, dysphagia and fonastenia.

Objective: the goal of this study is to evaluate prospectively the prevalence of compressive symptoms and their association with the volume of surgical specimens from a cohort of patients with goiter.

\footnotetext{
$1 \quad$ Estudiante de pregrado. Semillero de Investigación Scalpellum. Facultad de Medicina. Universidad de Cartagena. Cartagena. Colombia. Médico. Cirujano General. Grupo de Investigación Scalpellum. Profesor Asociado. Sección de Cirugía General. Facultad de Medicina. Universidad de Cartagena. Cartagena. Colombia. Cirujano de Cuello. E.S.E. Hospital Universitario del Caribe. Cartagena. Colombia.
} 
Methodology: through an interview in the preoperative act the manifestation of compressive symptoms by a cohort of patients with goiter were evaluated and subsequently, the volume of each surgical specimen was calculated. The association between the prevalence of compressive symptoms and its association with the volume of surgical specimens was determined.

Results: in this study participed 92 patients, $86(93.5 \%)$ women and $6(6.5 \%)$ men, with an average age of $46.1 \pm 14.9$ years. The prevalence symptoms were: dysphagia, 38 patients $(41.3 \%)$; orthopnea, 36 patients $(39.1 \%)$ and fonastenia, 11 patients $(12.0 \%)$. The absence of symptoms and its isolated finding were associated with volumes less than $50 \mathrm{~mL}(p=0.000)$. The simultaneous discovery of orthopnea and dysphagia associated with volumes between 50 and 99 milliliters $(p=0.000)$, finally finding fonastenia associated with dyspnea and dysphagia were associated with more than 100 milliliters $(p=0.000)$ volumes.

Conclusion: the surgical specimen volume is associated with the discovery of compressive symptoms in patients with goiter. The results of this study could be applied in the clinical setting in order to justify the intention to perform surgical procedures of compressive symptoms in the context of patients with goiter. Rev.cienc.biomed. 2015;6(2):259-264

\section{KEY WORDS}

Goiter; Signs; Symptoms.

\section{INTRODUCCIÓN}

La tiroides es una glándula endocrina de consistencia blanda, sus lóbulos miden en promedio 50 milímetros de diámetro longitudinal, 30 milímetros de diámetro transversal y 10 milímetros de diámetro anteroposterior (1). Su volumen y peso promedio son de 13 \pm 3.3 mililitros y 25 gramos en el varón (24 ) y de $7 \pm 1.7$ mililitros y 20 gramos, en la mujer (2-5).

La glándula tiroides está ubicada en la región anterior del cuello, envuelve posteriormente a la vía aerodigestiva superior, y se relaciona íntimamente con los nervios laríngeos recurrentes, desde su recorrido a lo largo del surco traqueoesofágico hasta su ingreso a la musculatura prelaríngea (6).

Se define como bocio al aumento en el peso de la glándula tiroides por encima de 20 gramos en la mujer, y 25 gramos en el varón, condición que genera un consecuente aumento patológico del volumen glandular (7). En virtud a que el cuello se considera la región anatómica con la mayor densidad de órganos y estructuras por área corporal, fenómenos patológicos como la bociogénesis y las enfermedades autoinmunes y neoplásicas de la glándula tiroides, son capaces de ocasionar un efecto de masa que trae consigo la manifestación clínica por parte del paciente de síntomas compresivos (SC) (8). Estos síntomas compresivos, ortopnea, disfagia y fonastenia son manifestados por entre un $13-52 \%$ de los pacientes con bocio (9-12).

La ortopnea es el SC más frecuente, es manifestada por un 20 - 56\% de los pacientes con bocio, quienes refieren una sensación de ahogamiento experimentada con el decúbito dorsal, la cual mejora cuando se sientan o adquieren una posición de Fowler (13). La disfagia es el segundo SC más frecuente, se atribuye a un desplazamiento esofágico posterior o lateral secundario al efecto de masa, este es manifestado por un $12-26 \%$ de los pacientes con bocio (14).

Por último, la fonastenia, referida como la sensación de "apagamiento de la voz", es la manifestación de la hipofunción de las cuerdas vocales secundaria a la neuropraxia de los nervios laríngeos recurrentes. La fonastenia es el SC menos manifestado por los pacientes, no supera una frecuencia del $12 \%$ (15). Se necesitan estudios que evalúen y describan si existe una asociación entre las características morfológicas del bocio, en especial su volumen y la frecuencia de SC. EI objetivo fue evaluar en pacientes con bocio la prevalencia de SC y su asociación con el volumen de los especímenes quirúrgicos.

\section{MATERIALES Y MÉTODOS}

El presente estudio contó con el aval del Comité de Ética de la E.S.E. Hospital Universitario del Caribe. Durante el período de 
tiempo comprendido entre mayo de 2014 y junio de 2015, un total de 92 pacientes fueron ingresados al estudio. En ninguno de los pacientes se evidenció ecográficamente un bocio con componente retroesternal.

Evaluación clínica: durante el intervalo de tiempo previo al inicio del procedimiento quirúrgico cada uno de los pacientes fue sometido a una entrevista en la cual un investigador evaluó la manifestación o no de SC. La presencia de ortopnea fue evaluada con la siguiente pregunta: ¿siente usted que respira con mayor dificultad cuando está acostado en comparación a cuando está sentado o de pie?, se consideró que existía ortopnea cuando la respuesta era afirmativa. La presencia de disfagia fue evaluada con la siguiente pregunta: ¿usted siente que traga con dificultad sus alimentos?, se consideró que existía disfagia cuando la respuesta era afirmativa. La presencia de fonastenia fue evaluada con la siguiente pregunta: ¿siente usted que su voz se apaga cuando habla y que debe descansar para recobrar la fuerza de su voz? Se consideró que existía fonastenia cuando la respuesta fue afirmativa. Cuando el paciente no respondió afirmativamente a ninguna de las preguntas fue considerado como asintomático.

Cálculo del volumen del espécimen quirúrgico: para determinar el volumen glandular se evaluó macroscópicamente cada uno de los especímenes quirúrgicos en el Departamento de Anatomía Patológica de la E.S.E. Hospital Universitario del Caribe. Previo al corte del espécimen fue medido el diámetro longitudinal, el diámetro transversal y el diámetro antero-posterior de cada lóbulo tiroideo. Posteriormente se realizó el corte de cada espécimen para verificar la distribución de las lesiones nodulares. En los casos en donde existía compromiso bilobular se calculó el volumen glandular a partir de la suma del volumen de ambos lóbulos. En los casos en donde existía una o varias lesiones unilobulares, independiente a que el espécimen corresponda a una tiroidectomía parcial o total, se calculó el volumen glandular a partir del lóbulo comprometido.

El volumen de los lóbulos tiroideos fue calculado mediante la fórmula establecida por
Shreck - Kollner, Muller y Grebe (16), basada en la determinación del volumen de una estructura elipsoide, de la siguiente forma: diámetro longitudinal $x$ diámetro transversal $x$ diámetro anteroposterior $x(n / 6)$. El volumen del istmo tiroideo, a menos que estuviera comprometido por una lesión nodular y sus diámetros fueran evaluables, fue asumido como de $0.14 \mathrm{ml}$ en mujeres y de 0.18 $\mathrm{ml}$ en hombres (17), dicho valor fue sumado al obtenido de acuerdo a lo definido anteriormente. Por último, el diagnóstico histológico fue realizado de acuerdo a la clasificación internacional de tumores propuesta por la Organización Mundial de la Salud (18).

Categorización de pacientes según síntomas y volumen de especímenes quirúrgicos: se establecieron los siguientes grupos de acuerdo a las características clínicas: Grupo 1 , pacientes asintomáticos o que manifestaron ortopnea, disfagia o fonastenia en forma aislada. Grupo 2, pacientes que manifestaron ortopnea y disfagia en forma simultánea. Grupo 3, pacientes que manifestaron fonastenia asociada a ortopnea y disfagia. De la misma forma, se establecieron los siguientes grupos de acuerdo al volumen de los especímenes quirúrgicos: Grupo $A$, especímenes con un volumen menor a 50 mililitros. Grupo $B$, especímenes con un volumen entre 50 y 100 mililitros. Grupo C, especímenes con un volumen mayor a 100 mililitros.

Análisis estadístico: se hallaron las medidas de tendencia central con sus respectivas medidas de dispersión para resumir las variables cuantitativas, de acuerdo al cumplimiento o no de los criterios de normalidad. Se realizaron tablas de frecuencia para resumir las variables cualitativas. Se realizaron pruebas de significación estadística para verificar la diferencia de proporciones en el contraste de variables cualitativas aplicando el modelo chi-cuadrado. Se consideraron estadísticamente significativos aquellos valores de $p$ inferiores a 0.05. Para estos cálculos se utilizó el paquete estadístico SPSS 22.0.

\section{RESULTADOS}

La población de estudio estuvo conformada por 92 pacientes, 86 (93.5\%) mujeres y 6 $(6.5 \%)$ varones, con una edad promedio de 
$46.1 \pm 14.9$ años. No se encontró asociación estadísticamente significativa entre los grupos etarios y la frecuencia de síntomas compresivos $(p=0.251)$.

Un total de 45 pacientes (48.9\%) manifestó SC durante la entrevista. La prevalencia de síntomas fue: disfagia en 38 pacientes $(41.3 \%)$, ortopnea en $36 \mathrm{pa}$ cientes $(39.1 \%)$ y fonastenia en 11 pacientes $(12.0 \%)$. La prevalencia de síntomas, aislados o asociados entre sí fue la siguiente: ortopnea aislada, 7 pacientes $(7.6 \%)$; disfagia aislada, 9 pacientes $(9.8 \%)$; ortopnea asociada a disfagia, 18 pacientes $(19.6 \%)$ y fonastenia asociada a ortopnea y disfagia 11 (12.0\%).

Se identificaron lesiones nodulares limitadas a un lóbulo en 55 pacientes $(59.8 \%)$, mientras que en 37 pacientes $(40.2 \%)$ las lesiones nodulares comprometían el parénquima tiroideo de ambos lóbulos. Una mayor proporción de pacientes con lesiones bilobulares manifestó SC en comparación al grupo de pacientes con lesión unilobulares $(62.1 \%$ vs $40 \% ; p=0.037)$. La distribución porcentual de los SC categorizados según el compromiso nodular del parénquima tiroideo se resume en la Tabla No 1.

La mediana de volumen de los especímenes quirúrgicos de los pacientes que manifestaron SC a la entrevista fue estadísticamente superior a la mediana de volumen de los especímenes quirúrgicos de los pacientes asintomáticos (64 mililitros vs 19.5 mililitros; $p=0.000)$.

La ausencia de síntomas y su hallazgo aislado se asociaron, indistintamente, con volúmenes menores a 50 mililitros $(p<0.001)$. El hallazgo simultáneo de ortopnea y disfagia se asoció a volúmenes entre 50 y 99 mililitros $(p<0.001)$, por último, el hallazgo de fonastenia asociada a disnea y disfagia se asoció a volúmenes superiores a 100 mililitros $(p<0.001)$. La asociación entre las características clínicas y el volumen de los especímenes quirúrgicos se presentan en la Tabla No 2.

La frecuencia de los diagnósticos histopatológicos fue la siguiente: bocio nodular
TABLA NO 1. DISTRIBUCIÓN PORCENTUAL DE LOS SINTOMAS COMPRESIVOS EN LA POBLACIÓN DE ESTUDIO CATEGORIZADOS SEGÚN EL COMPROMISO NODULAR DEL PARÉNQUIMA TIROIDEO

\begin{tabular}{|l|c|c|c|c|}
\hline \multicolumn{5}{|c|}{$\mathbf{n}(\%)$} \\
\hline Clínica & $\begin{array}{c}\text { Lesiones } \\
\text { nodulares } \\
\text { unilobulares }\end{array}$ & $\begin{array}{c}\text { Lesiones } \\
\text { nodulares } \\
\text { bilobulares }\end{array}$ & Total & valor p \\
\hline Asintomático & $33(35.9)$ & $14(15.2)$ & $\begin{array}{c}47 \\
(51.1)\end{array}$ & 0.037 \\
\hline $\begin{array}{l}\text { Ortopnea } \\
\text { aislada }\end{array}$ & $4(4.3)$ & $3(3.3)$ & $7(7.6)$ & 0.882 \\
\hline $\begin{array}{l}\text { Disfagia } \\
\text { aislada }\end{array}$ & $8(8.6)$ & $1(1.1)$ & $9(9.7)$ & 0.061 \\
\hline $\begin{array}{l}\text { Ortopnea }+ \\
\text { Disfagia }\end{array}$ & $7(7.7)$ & $11(11.9)$ & $\begin{array}{c}18 \\
(19.6)\end{array}$ & 0.044 \\
\hline $\begin{array}{l}\text { Ortopnea }+ \\
\text { Disfagia }+ \\
\text { Fonastenia }\end{array}$ & $3(3.3)$ & $8(8.7)$ & $\begin{array}{c}11 \\
(12.0)\end{array}$ & 0.019 \\
\hline Total & $55(59.8)$ & $37(40.2)$ & $\begin{array}{c}92 \\
(100)\end{array}$ & - \\
\hline
\end{tabular}

\begin{tabular}{|c|c|c|c|c|c|}
\hline \multicolumn{6}{|c|}{$\begin{array}{c}\text { TABLA No } 2 . \\
\text { ASOCIACIÓN ENTRE LAS CAR } \\
\text { CLÍNICAS Y EL VOLUMEN DE LO } \\
\text { QUIRÚRGICOS } \\
n(\%)\end{array}$} \\
\hline \multirow{2}{*}{$\begin{array}{c}\text { Características } \\
\text { clínica }\end{array}$} & \multicolumn{3}{|c|}{$\begin{array}{c}\text { Volumen espécimen } \\
\text { quirúrgico }\end{array}$} & \multirow[b]{2}{*}{ Total } & \multirow[b]{2}{*}{ valor $\mathrm{p}$} \\
\hline & $\begin{array}{c}\text { Grupo } \\
\text { A } \\
\end{array}$ & $\begin{array}{c}\text { Grupo } \\
\text { B }\end{array}$ & $\begin{array}{c}\text { Grupo } \\
\text { C } \\
\end{array}$ & & \\
\hline Grupo 1 & $\begin{array}{c}60 \\
(65.2) \\
\end{array}$ & $\begin{array}{c}3 \\
(3.3) \\
\end{array}$ & $\begin{array}{c}0 \\
(0.0) \\
\end{array}$ & $\begin{array}{c}63 \\
(68.5) \\
\end{array}$ & $<0.0001$ \\
\hline Grupo 2 & $\begin{array}{c}2 \\
(2.2) \\
\end{array}$ & $\begin{array}{c}13 \\
(14.0) \\
\end{array}$ & $\begin{array}{c}3 \\
(3.3) \\
\end{array}$ & $\begin{array}{c}18 \\
(19.5) \\
\end{array}$ & $<0.001$ \\
\hline Grupo 3 & $\begin{array}{c}1 \\
(1.1) \\
\end{array}$ & $\begin{array}{c}3 \\
(3.3) \\
\end{array}$ & $\begin{array}{c}7 \\
(7.6) \\
\end{array}$ & $\begin{array}{c}11 \\
(12.0) \\
\end{array}$ & $<0.001$ \\
\hline Total & $\begin{array}{c}63 \\
(68.5) \\
\end{array}$ & $\begin{array}{c}19 \\
(20.6) \\
\end{array}$ & $\begin{array}{c}10 \\
(10.9)\end{array}$ & $\begin{array}{c}92 \\
(100.0) \\
\end{array}$ & - \\
\hline
\end{tabular}

simple, 34 (37.0\%); bocio multinodular simple, 29 (31.5\%); adenoma folicular, $11(12.0 \%)$; enfermedad de Hashimoto, 8 $(8.7 \%)$; carcinoma papilar, $8(8.7 \%)$; carcinoma folicular, $1(1.1 \%)$; y carcinoma medular, $1(1.1 \%)$. Se encontró una menor prevalencia de SC en pacientes con bocio nodular simple $(p=0,001)$, mientras que en grupo de pacientes con bocio multinodular simple se encontró una mayor prevalencia de SC $(p=0,000)$. La asociación entre los diagnósticos histológicos y la frecuencia de SC se muestra en la Tabla N³. 


\begin{tabular}{|l|c|c|c|c|c|c|c|}
\hline \multicolumn{7}{|c|}{ TABLA NO 3. } \\
SINTOMAS COMPRESIVOS Y ASOCIACIÓN CON LOS DIAGNÓSTICOS HISTOPATOLÓGICOS \\
\hline & BNS & BMS & EH & AF & CP & CF & CM \\
\hline Asintomático & $26(76.5)$ & $4(13.8)$ & $5(62.5)$ & $5(45.5)$ & $7(87.5)$ & $0(0.0)$ & $0(0.0)$ \\
\hline Ortopnea aislada & $2(5.9)$ & $5(17.2)$ & $0(0.0)$ & $0(0.0)$ & $0(0.0)$ & $0(0.0)$ & $0(0.0)$ \\
\hline Disfagia aislada & $4(11.8)$ & $2(6.9)$ & $0(0.0)$ & $2(18.2)$ & $1(12.5)$ & $0(0.0)$ & $0(0.0)$ \\
\hline Ortopnea + Disfagia & $2(5.9)$ & $10(34.5)$ & $1(12.5)$ & $3(27.3)$ & $0(0.0)$ & $1(100.0)$ & $1(100.0)$ \\
\hline $\begin{array}{l}\text { Ortopnea + Disfagia + } \\
\text { Fonastenia }\end{array}$ & $0(0.0)$ & $8(27.6)$ & $2(25.0)$ & $1(9.9)$ & $0(0.0)$ & $0(0.0)$ & $0(0.0)$ \\
\hline Total & $34(100.0)$ & $29(100.0)$ & $8(100.0)$ & $11(100.0)$ & $8(100.0)$ & $1(100.0)$ & $1(100.0)$ \\
\hline p valor & 0.001 & 0.000 & 0.522 & 0.664 & 0.217 & 0.385 & 0.385 \\
\hline
\end{tabular}

BNS: bocio nodular simple; BMS: bocio multinodular simple; EH: Enfermedad de Hashimoto; AF: adenoma folicular; CP: carcinoma papilar; CF: carcinoma folicular; CM: carcinoma medular.

\section{DISCUSIÓN}

Se presentó un estudio de linealidad prospectiva, con el cual se intentó identificar la posible asociación entre el volumen del espécimen quirúrgico y la manifestación de SC. La disfagia fue el SC manifestado con mayor frecuencia por los pacientes, mostrando una prevalencia del $41 \%$, seguido por la ortopnea con una prevalencia del $39,1 \%$, mientras que la fonastenia mostró una prevalencia acorde con la literatura, consistente en un $12 \%$.

Resulta lógico pensar que la mayoría de especímenes con lesiones unilobulares de volúmenes menores a $50 \mathrm{ml}$ no generen síntomas $(p=0.037)$, pero el análisis estadístico sugirió diversos comportamientos clínicos. Una mayor proporción de pacientes que manifestaron ortopnea o disfagia en forma aislada poseían un espécimen quirúrgico con un volumen menor a $50 \mathrm{ml}(p=0.000)$, en este grupo de pacientes llama la atención que la distribución de las lesiones nodulares en el parénquima tiroideo no se asoció con una mayor frecuencia de ortopnea $(p=0.882)$, mientras que una mayor proporción de pacientes con lesiones nodulares limitadas a un único lóbulo tiroideo manifestó en mayor medida disfagia $(p=0.062)$.

Lo anterior sugiere que en el caso de los pacientes con ortopnea, este síntoma puede ser secundario a otros factores que el efecto de masa producido por un único lóbulo tiroideo, pese a su pequeño volumen, sí podría generar un desplazamiento esofágico lateral y responder a la disfagia manifestada por este grupo de pacientes. Un gran número de pacientes con lesiones nodulares bilobulares manifestó con mayor frecuencia ortopnea y disfagia en forma asociada $(p=0,044)$, además, cuando los especímenes quirúrgicos poseían un volumen entre $50 \mathrm{ml}$ y $90 \mathrm{ml}$ $(p=0.000)$. Se observó que dos especímenes con volúmenes menores a $50 \mathrm{ml}$ y tres especímenes con volúmenes mayores a $100 \mathrm{ml}$ manifestaron ortopnea y disfagia en forma asociada, lo anterior podría responder a la gran diferencia en la anatomía cervical que existe de un individuo a otro, y su interacción con la glándula tiroides bocígena (19).

La fonastenia parece asociarse, en mayor proporción, a un síntoma compresivo cuando el volumen del espécimen quirúrgico supera los $100 \mathrm{ml}$, y no fue manifestado aisladamente por ningún paciente. Por último, en los pacientes cuyo espécimen quirúrgico fue menor a $100 \mathrm{ml}$ y manifestaron fonastenia asociada a disfagia y ortopnea, puede estar asociado a un componente meramente anatómico (19).

Se encontró asociación estadísticamente significativa entre la manifestación de SC 
y el diagnóstico histopatológico $(p=0.001)$, dicha asociación responde a dos fenómenos: en primer lugar, una gran proporción de pacientes con bocio nodular simple fueron asintomáticos $(p=0.001) y$, en segundo lugar, los pacientes con bocio multilocular simple presentaban en mayor proporción SC $(p=0.000)$. Por lo tanto, en virtud a la distribución homogénea de SC en el resto de diagnósticos histológicos, los datos reflejan que no existe relación entre estos y presencia de síntomas.

\section{CONCLUSIÓN}

Se reportó una prevalencia de disfagia, ortopnea y fonastenia del $41.3 \%, 39.1 \%$ y $12.0 \%$, respectivamente. Los resultados sugieren que el volumen del espécimen quirúrgico está asociado con el hallazgo de SC en el paciente con bocio. El estudio se respaldó en la aplicabilidad aislada de la anamnesis como una herramienta en la exploración clínica de SC en pacientes con bocio. Se precisa realizar estudios en los cuales se comparen estos hallazgos con la evaluación objetiva de SC mediante la utilización de estudios endoscópicos como esofagoscopia y la fibrolaringoscopia.

\section{CONFLICTOS DE INTERESES: ninguno} que declarar.

FINANCIACIÓN: recursos propios de los autores.

\section{REFERENCIAS BIBLIOGRÁFICAS}

1. Aydıner Ö, Karakoç Aydıner E, Akpınar I, Turan S, Bereket A. Normative data of thyroid volume-ultrasonografic evaluation of 422 subjects aged 0-55 years. J Clin Res Pediatr Endocrinol. $2015 ; 7(2): 98-101$.

2. Oberhofer R, Ober A, Oberkofler F, Amor H. Thyroid gland volumes of healthy adults in an area with endemic goiter. Acta Med Austrica. 1989;16(2):38-41.

3. Gomez JM, Maravall FJ, Gómez N, Gumà A, Casamitjana R, Soler J. Pituitary-thyroid axis, thyroid volume and leptin in healthy adults. Horm Metab Res. 2002;34(2):67-71.

4. Noa SS, Cantillo M, Quintana N. El volumen tiroideo por ultrasonido en población masculina adulta sana de Ciego de Avila. MediCiego. 2013;19(2):13-9.

5. Noa SS, Cantillo M, Quintana N. El volumen tiroideo por ultrasonido en mujeres sana avileñas. MediCiego. 2013;19(Supl1).

6. Testut L, Latarjet A. Glándulas de secreción interna o glándulas endocrinas. Artículo primero: Cuerpo tiroides. En: Tratado de Anatomía Humana. 9na ed. Barcelona: Salvat Editores S.A. 1983;3:1025-51.

7. Ardila E, Escobar ID, Páez A. Asociación Colombiana de Endocrinología: Consenso Colombiano para el diagnóstico y manejo de las enfermedades tiroideas. Acta Med Colomb. 1998;24(4):15974.

8. Chen AY, Bernet VJ, Carty SE, Davies TF, Ganly I, Inabet WB 3rd, et al. American Thyroid Association Statement on optimal surgical management of goiter. Thyroid. 2014;24(2):181-9.

9. Pinchera A, Aghini-Lombardi F, Antonangeli L, Vitti P. Multinodular goiter. Epidemiology and prevention. Ann Ital Chir. 1996;67(3):317-25.

10. Wong CKM, Wheeler MH. Thyroid nodules: rational management, World J Surg. 2000;24:93441.

11. Banks CA, Ayers CM, Hornig JD, Lentchs MD, Day TA, Nguyen SA, et al. Thyroid disease and compressive symptom. Laryngoscope 2011, 122:13-6

12. Pereira JA, Girvent M, Sancho JJ, Parada C, Sitges-Sierra A. Prevalence of long.tem upper aerodigestive symptoms after uncomplicated bilateral thyroidectomy. Surgery. 2003;133:318-22.

13. Torchio R, Gulotta C, Perboni A, Ciacco C, Guglielmo M, Orlandi F, et al. Orthopnea and tidal expiratory flow limitation in patients with euthyroid goiter. Chest. 2003;124:133-40.

14. Ingbar SH, Woeber KA Diseases of the thyroid. In: Petersdorf RG, et al., eds.Harrison's Principles of Internal Medicine. New York: McGraw-Hill, 1983;614-34.

15. Cornut G, Bouchayer M. From the functional to the organic disorders in phoniatrics. Rev Laryngol Otol Rhinol (Bord). 1987;108:417-9.

16. Sherk-Koller $H$, Muller $H$, Grebe SF. Ultraschalldiagnostik der Schilddrusse. Radiologe. $1984 ; 24: 461-7$.

17. Chang $\mathrm{CY}$, Lei YF, Tseng $\mathrm{CH}$, Shih SR. Thyroid segmentation and volume estimation in ultrasound images. IEEE Trans Biomed Eng. 2010; 57(6):1348-57.

18. Organización Mundial de la Salud. Clasificación internacional de tumores: tipos histológicos de tumores tiroideos. Ginebra: OMS; 1974.

19. Sanabria Á, Chala A, Ramírez A, Álvarez A. Anatomía quirúrgica cervical de importancia en cirugía tiroidea. Rev Colomb Cir. 2014;29(1):40-8. 